\title{
Idempotents for Birman-Murakami-Wenzl algebras and reflection equation
}

\author{
A. P. Isaev, A. I. Molev and O. V. Ogievetsky ${ }^{1}$
}

\begin{abstract}
A complete system of pairwise orthogonal minimal idempotents for Birman-Murakami-Wenzl algebras is obtained by a consecutive evaluation of a rational function in several variables on sequences of quantum contents of up-down tableaux. A by-product of the construction is a one-parameter family of fusion procedures for Hecke algebras. Classical limits to two different fusion procedures for Brauer algebras are described.
\end{abstract}

\section{Introduction}

Let $V$ be a vector space equipped with a non-degenerate bilinear (symmetric or antisymmetric) form. Let $G$ be the group of linear transformations of $V$ preserving the form. The Brauer-Schur-Weyl duality relates tensor representations of $G$ with representations of the Brauer algebras. The "quantum group" version of the Brauer-Schur-Weyl duality relates tensor representations of a quantum group associated with $G$ with representations of the Birman-Murakami-Wenzl (BMW) algebras, introduced in [2] and [28]. A quantum group associated with $G$ can be defined through an $R$-matrix of the "BCD-type"; it is an endomorphism of $V \otimes V$, having three eigenvalues with prescribed multiplicities, which satisfies the Yang-Baxter equation together with extra conditions ensuring that the tensor powers of $V$ carry a "local" representation of the tower of the BMW algebras. Thus, in contrast to the classical situation, the quantum version of the Brauer-Schur-Weyl duality requires a choice of a $B C D$-type $R$-matrix. It should be noted that the problem of a description of $B C D$-type $R$-matrices is difficult and far from being completed (it is an open question already for $R$-matrices of the $A$-type, see [32]). To use the quantum version of the Brauer-Schur-Weyl

\footnotetext{
${ }^{1}$ On leave of absence from P. N. Lebedev Physical Institute, Leninsky Pr. 53, 117924 Moscow, Russia.
} 
duality one needs to know explicit bases in the representation spaces of the BMW algebras. This task splits into two parts. First, one needs to know a complete set of pairwise orthogonal minimal idempotents of the BMW algebras; second, to calculate, given a $B C D$-type $R$-matrix, the image of these idempotents in the corresponding local representation. The present paper is a contribution to the first, $R$-matrix independent, part of the task, it deals with formulas for minimal idempotents of the BMW algebras. Such formulas can be written down in terms of the generators of the BMW algebras, without a concrete choice of an $R$-matrix. This requires a good understanding of the representation theory of the BMW algebras. Different aspects of the representation theory of the BMW algebras were discussed in the literature, see e.g. [1], [2], [23], [35] and [37]. The family of the BMW-algebras depends on a discrete parameter $n=0,1,2, \ldots$ and two continuos parameters, $q$ and $\nu$. Formulas for the action of the generators of the BMW-algebras on spaces of their irreducible representations were given in [28]. The eigenvalues of the so called Jucys-Murphy elements were calculated in [23], see also [17] (the article [23] does not use terminology "Jucys-Murphy elements"). One can show that the Jucys-Murphy elements generate the maximal commutative subalgebra of the BMW algebra; in particular, the Jucys-Murphy elements distinguish the basis vectors and thus imply formulas for the complete set of pairwise orthogonal minimal idempotents.

We suggest another formula for the same set of idempotents in the present paper. Our way to describe the idempotents is in the spirit of what is often referred to as the fusion procedure. For generic values of the parameters of the BMW algebra, basis vectors in the irreducible representations are in one-to-one correspondence with sequences of quantum contents, see precise definitions in Section 3. We introduce in Section 3 a certain BMW algebra-valued rational function in several variables (which we call fusion function). The idempotent, corresponding to a given content sequence, is then obtained by a consecutive evaluation of the fusion function at values of these variables given by the quantum contents. The fusion procedure (for the symmetric group) originates in the work of Jucys [19], see also [5] and [30]. A version of the fusion procedure for the symmetric group involving the consecutive evaluation appeared in [25]. The fusion function was written down for the Hecke algebras and Brauer algebras, see [11], [12], [13] and [31].

The BMW algebra admits a natural quotient isomorphic to the Hecke algebra. The BMW algebra depends on a certain parameter $\nu$, but this dependence disappears in the Hecke algebra quotient. Nevertheless our fusion function for the BMW algebra depends on $\nu$ non-trivially. As a consequence, 
the image of the BMW fusion function in the Hecke algebra quotient provides a one parameter family, see paragraph 5 of Section 3 , of the fusion functions for the Hecke algebras.

In paragraph 6 Section 3 we make an observation on a deep relation between the BMW fusion function and the solution, presented in [15], of the so called reflection equation. Thus for algebras related to the centralizer construction for classical and quantum groups of types $G L, O$ and $S p$ (and $O S p$ for super-groups) in the defining representation (like the group algebra of the symmetric group, the Hecke algebra, the Brauer algebra and the Birman-Murakami-Wenzl algebra) the fusion function is built with the help of solutions of the reflection equation.

The classical version of the reflection equation was used in [12] to construct certain evaluation homomorphisms. We postpone the study of the evaluation homomorphisms for the quantum algebras for a future work.

Two different versions of the fusion functions for the Brauer algebra are given in [11] and [12]. The Brauer algebra can be obtained in several ways as a contraction (or classical limit) of the BMW algebra. In Section 4 we derive both fusion functions for the Brauer algebra from the same fusion function for the BMW algebra using two different contractions.

\section{Birman-Murakami-Wenzl algebra}

\subsection{Definition and basic relations}

The Birman-Murakami-Wenzl algebra $B M W_{n}(q, \nu)$ was defined in [2], [26] and [28]. It is generated over $\mathbb{C}$ by invertible elements $T_{1}, \ldots, T_{n-1}$ with the following defining relations

$$
\begin{gathered}
T_{i} T_{i+1} T_{i}=T_{i+1} T_{i} T_{i+1}, \quad T_{i} T_{j}=T_{j} T_{i} \text { for }|i-j|>1, \\
\kappa_{i} T_{i}=T_{i} \kappa_{i}=\nu \kappa_{i}, \\
\kappa_{i} T_{i-1}^{\varepsilon} \kappa_{i}=\nu^{-\varepsilon} \kappa_{i}, \quad \kappa_{i} T_{i+1}^{\varepsilon} \kappa_{i}=\nu^{-\varepsilon} \kappa_{i} \text { with } \varepsilon= \pm 1,
\end{gathered}
$$

where

$$
\kappa_{i}:=1-\frac{T_{i}-T_{i}^{-1}}{q-q^{-1}} .
$$


Here $q$ and $\nu$ are complex parameters of the algebra which we assume generic in the sequel; in particular, the definition (2.4) makes sense, the denominator in the right hand side does not vanish.

The quotient of the algebra $B M W_{n}(q, \nu)$ by the ideal generated by the elements $\kappa_{1}, \ldots, \kappa_{n}$ (in fact, this ideal is generated by any one of these elements, say, $\left.\kappa_{1}\right)$ is isomorphic to the Hecke algebra $H_{n}(q)$. We shall often omit the parameters in the notation for the algebras and write simply $B M W_{n}$ and $H_{n}$.

Let

$$
\mu=\frac{q-q^{-1}+\nu^{-1}-\nu}{q-q^{-1}}=\frac{\left(q^{-1}+\nu\right)(q-\nu)}{\nu\left(q-q^{-1}\right)} .
$$

The following relations can be derived from (2.1)-(2.3):

$$
\kappa_{i}^{2}=\mu \kappa_{i}
$$

then, with $\varepsilon= \pm 1$,

$$
\begin{aligned}
\kappa_{i} T_{i+\varepsilon} T_{i} & =T_{i+\varepsilon} T_{i} \kappa_{i+\varepsilon} \\
\kappa_{i} \kappa_{i+\varepsilon} \kappa_{i} & =\kappa_{i} \\
\left(T_{i}-\left(q-q^{-1}\right)\right) \kappa_{i+\varepsilon}\left(T_{i}\right. & \left.-\left(q-q^{-1}\right)\right) \\
& =\left(T_{i+\varepsilon}-\left(q-q^{-1}\right)\right) \kappa_{i}\left(T_{i+\varepsilon}-\left(q-q^{-1}\right)\right) \\
T_{i+\varepsilon} \kappa_{i} T_{i+\varepsilon} & =T_{i}^{-1} \kappa_{i+\varepsilon} T_{i}^{-1}
\end{aligned}
$$

and

$$
\begin{aligned}
\kappa_{i} T_{i+\varepsilon} T_{i} & =\kappa_{i} \kappa_{i+\varepsilon}, \\
\kappa_{i} T_{i+\varepsilon}^{-1} T_{i}^{-1} & =\kappa_{i} \kappa_{i+\varepsilon} \\
\kappa_{i+\varepsilon} \kappa_{i}\left(T_{i+\varepsilon}-\left(q-q^{-1}\right)\right) & =\kappa_{i+\varepsilon}\left(T_{i}-\left(q-q^{-1}\right)\right),
\end{aligned}
$$

together with their images under the anti-automorphism $\rho$ of the algebra $B M W_{n}$ defined on the generators by

$$
\rho\left(T_{i}\right)=T_{i} .
$$




\subsection{Baxterized elements}

The baxterized elements $T_{i}(u, v)$ are defined by

$$
T_{i}(u, v):=T_{i}+\frac{q-q^{-1}}{v / u-1}+\frac{q-q^{-1}}{1+\nu^{-1} q v / u} \kappa_{i}
$$

see [3], [10], [18] and [27]. They are rational functions in complex variables $u$ and $v$ which are called spectral variables. The elements $T_{i}(u, v)$ depend on the ratio of the spectral parameters; later we shall evaluate spectral variables $u$ and $v$ in terms of contents of boxes of Young diagram; this explains the use of having both spectral variables in the notation (2.15) for the baxterized element. However for brevity we shall denote sometimes the baxterized elements by $T_{i}(u / v)$ (with one argument only), $T_{i}(u):=T_{i}(u, 1)$.

The baxterized elements satisfy the braid relation of the form

$$
\begin{aligned}
& T_{i}\left(u_{2}, u_{3}\right) T_{i+1}\left(u_{1}, u_{3}\right) T_{i}\left(u_{1}, u_{2}\right) \\
& =T_{i+1}\left(u_{1}, u_{2}\right) T_{i}\left(u_{1}, u_{3}\right) T_{i+1}\left(u_{2}, u_{3}\right) .
\end{aligned}
$$

The inverses of the baxterized elements are given by

$$
T_{i}(v, u)^{-1}=T_{i}(u, v) f(u, v)
$$

where

$$
f(u, v)=\frac{(u-v)^{2}}{\left(u-q^{2} v\right)\left(u-q^{-2} v\right)}=f(v, u) .
$$

Let

$$
Q_{i}(u, v ; c):=T_{i}\left(\frac{1}{c u v}\right)=T_{i}+\frac{q-q^{-1}}{c u v-1}+\frac{q-q^{-1}}{1+\nu^{-1} q c u v} \kappa_{i}
$$

We keep three arguments $c, u$ and $v$ for the later convenience.

It follows from (2.16) that

$$
\begin{aligned}
T_{i}\left(u_{2}, u_{3}\right) Q_{i+1}\left(u_{1}, u_{3} ; c\right) Q_{i}\left(u_{1}, u_{2} ; c\right) \\
=Q_{i+1}\left(u_{1}, u_{2} ; c\right) Q_{i}\left(u_{1}, u_{3} ; c\right) T_{i+1}\left(u_{2}, u_{3}\right)
\end{aligned}
$$




\subsection{Jucys-Murphy elements}

The Jucys-Murphy elements of the algebra $B M W_{n}$ are defined by

$$
y_{1}=1, \quad y_{k+1}=T_{k} \ldots T_{2} T_{1}^{2} T_{2} \ldots T_{k}, \quad k=1, \ldots, n-1
$$

The elements $y_{1}, \ldots, y_{n}$ pairwise commute and satisfy the identities

$$
\kappa_{j} y_{j+1} y_{j}=y_{j} y_{j+1} \kappa_{j}=\nu^{2} \kappa_{j}
$$

The Jucys-Murphy elements were originally used for constructing idempotents for the symmetric groups in [18], [27]. Analogues of the JucysMurphy elements can be defined for a number of important algebras related to the symmetric group rings; they turn out to generate maximal commutative subalgebras in these rings (see [12], [14], [33], [34] and references therein). The commutative subalgebra, generated by the Jucys-Murphy elements $y_{1}, \ldots, y_{n}$, of the generic algebra $B M W_{n}$ is maximal as well; it follows from the results in [17],[23]. In the next section we use this maximality for the construction of the idempotents for the BMW algebra.

\section{Idempotents for BMW algebra}

\subsection{Up-down tableaux}

Recall that the Bratteli diagram for the chain of generic BMW algebras is the up-down Young graph; thus the irreducible representations of the generic algebra $B M W_{n}$ are in one-to-one correspondence with the partitions $\lambda$ of $n-2 k, 0 \leqslant k \leqslant\lfloor n / 2\rfloor$. We use the convention to depict a partition $\lambda=\left(\lambda_{1}, \lambda_{2}, \ldots\right)$ by its Young diagram which is the left-justified array of rows of boxes containing $\lambda_{1}$ boxes in the first row, $\lambda_{2}$ boxes in the second row and so on. Basis vectors $v_{U_{n}}$ in the irreducible representation corresponding to $\lambda$ are indexed by the up-down tableaux of shape $\lambda$, that is, by sequences $U_{n}=\left(\Lambda_{1}, \Lambda_{2}, \ldots, \Lambda_{n}\right)$ of Young diagrams, such that $\Lambda_{1}=\square, \Lambda_{j}$ differs from $\Lambda_{j-1}$ by exactly one box (added or removed), $j=2, \ldots, n$, and $\Lambda_{n}=\lambda$; see [8] for necessary definitions of Bratteli diagrams etc.

The usual notion of transposition of Young diagrams and tableaux extends to up-down tableaux; for an up-down tableau $U_{n}=\left(\Lambda_{1}, \Lambda_{2}, \ldots, \Lambda_{n}\right)$ the sequence $\left(\Lambda_{1}^{t}, \Lambda_{2}^{t}, \ldots, \Lambda_{n}^{t}\right)$, formed by the transposed Young diagrams, is clearly an up-down tableau and we set $U_{n}^{t}:=\left(\Lambda_{1}^{t}, \Lambda_{2}^{t}, \ldots, \Lambda_{n}^{t}\right)$. 
The up-down Young graph arises in the study of the common spectrum of the Jucys-Murphy elements within the algebra itself [17]; as for representations, the images of the Jucys-Murphy elements are diagonal in the chosen basis,

$$
\rho_{\lambda}\left(y_{j}\right) v_{U_{n}}=c_{j}\left(U_{n}\right) v_{U_{n}}
$$

Here $v_{U_{n}}$ is the basis vector associated to the up-down tableau $U_{n}=\left(\Lambda_{1}, \Lambda_{2}\right.$, $\left.\ldots, \Lambda_{n}\right)$ of shape $\lambda$ and $\rho_{\lambda}(z)$ is the operator of the element $z \in B M W_{n}$ in the representation corresponding to $\lambda$; the number $c_{j}\left(U_{n}\right)$ is the quantum content of the box added/removed at the step $j$ of building the up-down tableau $U_{n}$; for the box in the $a$-th row and $b$-th column the quantum content is defined by

$$
c_{j}\left(U_{n}\right)=q^{2(b-a)}
$$

if this box was added to the diagram $\Lambda_{j-1}$ to obtain the diagram $\Lambda_{j}$ and

$$
c_{j}\left(U_{n}\right)=\nu^{2} q^{2(-b+a)}
$$

if this box was removed from the diagram $\Lambda_{j-1}$ to obtain the diagram $\Lambda_{j}$. The spectrum of the Jucys-Murphy elements in the irreducible representations of the BMW algebra was calculated in [23].

Note that $c_{1}=1$. In the generic regime, the up-down tableau $U_{n}$ is uniquely determined by its content sequence $\left\{c_{1}\left(U_{n}\right), \ldots, c_{n}\left(U_{n}\right)\right\}$.

We denote by $E_{U_{n}} \equiv E_{U_{n}}^{\lambda} \in B M W_{n}$ the primitive idempotent related to the vector $v_{U_{n}}$ in the irreducible representation corresponding to a diagram $\lambda$. That is, $E_{U_{n}}$ is the projector onto the 1-dimensional subspace spanned by the vector $v_{U_{n}}$ along the subspace spanned by the other basis vectors, and $E_{U_{n}}$ annihilates all other irreducible representations.

\subsection{Inductive formula for idempotents of $\mathrm{BMW}$ algebras}

For an up-down tableau $U_{n}=\left(\Lambda_{1}, \Lambda_{2}, \ldots, \Lambda_{n-1}, \Lambda_{n}\right)$ of length $n$ we denote by $U_{k}, k \leqslant n$, the initial segment of $U_{n}$ of length $k, U_{k}:=\left(\Lambda_{1}, \Lambda_{2}, \ldots, \Lambda_{k}\right)$. For brevity, let $c_{j}=c_{j}\left(U_{n}\right)$ and let $c$ be a parameter, $c \neq c_{n}^{-2}$. Note that by (3.2) the Jucys-Murphy element $y_{n}$ takes the value $c_{n}$ on the image of the idempotent $E_{U_{n}}$. Suppose that $Y$ is an eigenvalue of $y_{n}$ on the image of $E_{U_{n-1}}$. For $Y \neq c_{n}$ the rational function $\frac{(c u Y-1)}{(u-Y)} \frac{\left(u-c_{n}\right)}{\left(c u c_{n}-1\right)}$ vanishes at $u=c_{n}$ 
while for $Y=c_{n}$ this rational function simplifies to 1. Thus,

$$
E_{U_{n}}=\left.E_{U_{n-1}} \frac{\left(c u y_{n}-1\right)}{\left(u-y_{n}\right)} \frac{\left(u-c_{n}\right)}{\left(c u c_{n}-1\right)}\right|_{u=c_{n}}
$$

with arbitrary $c \neq c_{n}^{-2}$. The parameter $c$ will be fixed below:

$$
c=-q^{-1} \nu^{-1}
$$

The form of the rational function in the right hand side of (3.4) and the choice (3.5) of $c$ are suggested by the proof of Lemma 3.1. With the choice (3.5) the inequality $c \neq c_{n}^{-2}$ holds in the generic regime.

\subsection{Preparatory lemma}

We shall rewrite the product $E_{U_{n-1}} \frac{c u y_{n}-1}{u-y_{n}}$ in the right hand side of (3.4). Define a family of $B M W_{n}$-valued rational functions by

$$
\mathcal{Y}_{1}(u):=\frac{c u y_{1}-1}{u-y_{1}}
$$

and, for $j=2, \ldots, n$,

$$
\begin{aligned}
\mathcal{Y}_{j}\left(u_{1}, \ldots, u_{j}\right) & \\
:= & Q_{j-1}\left(u_{j-1}, u_{j} ; c\right) \mathcal{Y}_{j-1}\left(u_{1}, \ldots, u_{j-2}, u_{j}\right) T_{j-1}\left(u_{j}, u_{j-1}\right)^{-1} \\
= & Q_{j-1}\left(u_{j-1}, u_{j} ; c\right) \cdots Q_{1}\left(u_{1}, u_{j} ; c\right) \mathcal{Y}_{1}\left(u_{j}\right) T_{1}^{-1}\left(u_{j}, u_{1}\right) \\
& \cdots T_{j-1}\left(u_{j}, u_{j-1}\right)^{-1}
\end{aligned}
$$

\section{Lemma 3.1.}

$$
E_{U_{n-1}} \mathcal{Y}_{n}\left(c_{1}, c_{2}, \ldots, c_{n-1}, u\right)=E_{U_{n-1}} \frac{c u y_{n}-1}{u-y_{n}}
$$

Proof. We prove (3.8) by induction on $n$. The induction base is $n=1\left(E_{U_{0}}=\right.$ $1, U_{0}$ is empty) is tautological. By (3.7) and the induction hypothesis, (3.8) reduces to the equality

$$
E_{U_{n-1}} Q_{n-1}\left(c_{n-1}, u ; c\right) \frac{c u y_{n-1}-1}{u-y_{n-1}} T_{n-1}\left(u, c_{n-1}\right)^{-1}=E_{U_{n-1}} \frac{c u y_{n}-1}{u-y_{n}} .
$$


Since $y_{n}$ commutes with $E_{U_{n-1}}$, this can be rewritten in the form

$$
\begin{aligned}
& E_{U_{n-1}}\left(u-y_{n}\right) Q_{n-1}\left(c_{n-1}, u ; c\right)\left(c u y_{n-1}-1\right) \\
& =E_{U_{n-1}}\left(c u y_{n}-1\right) T_{n-1}\left(u, c_{n-1}\right)\left(u-y_{n-1}\right)
\end{aligned}
$$

or, with the definition (2.19),

$$
\begin{aligned}
& E_{U_{n-1}}\left(u-y_{n}\right)\left(T_{n-1}+\frac{q-q^{-1}}{c c_{n-1} u-1}+\frac{q-q^{-1}}{1+\nu^{-1} q c c_{n-1} u} \kappa_{n-1}\right)\left(c u y_{n-1}-1\right) \\
= & E_{U_{n-1}}\left(c u y_{n}-1\right)\left(T_{n-1}+\frac{\left(q-q^{-1}\right) u}{c_{n-1}-u}+\frac{\left(q-q^{-1}\right) u}{u+\nu^{-1} q c_{n-1}} \kappa_{n-1}\right)\left(u-y_{n-1}\right) .
\end{aligned}
$$

We transform the left hand side of (3.11) to

$$
\begin{aligned}
E_{U_{n-1}}\left(u-y_{n}\right)( & \left.\operatorname{cuy}_{n} \underline{\left(T_{n-1}-\left(q-q^{-1}\right)\right.}+\left(q-q^{-1}\right) \kappa_{n-1}\right) \\
& -\underline{T_{n-1}}+\frac{q-q^{-1}}{c c_{n-1} u-1} \frac{\left(c u c_{n-1}-1\right)}{} \\
& \left.+\frac{q-q^{-1}}{1+\nu^{-1} q c c_{n-1} u} \kappa_{n-1}\left(\text { cu } y_{n-1}-1\right)\right)
\end{aligned}
$$

and the right hand side of (3.11) to

$$
\begin{aligned}
E_{U_{n-1}}\left(c u y_{n}-1\right)( & -y_{n}\left(\underline{T_{n-1}-\left(q-q^{-1}\right)}+\left(q-q^{-1}\right) \kappa_{n-1}\right) \\
& +\underline{u T_{n-1}}+\frac{\left(q-q^{-1}\right) u}{c_{n-1}-u} \underline{\left(u-c_{n-1}\right)} \\
& \left.+\frac{\left(q-q^{-1}\right) u}{u+\nu^{-1} q c_{n-1}} \kappa_{n-1}\left(u-y_{n-1}\right)\right) .
\end{aligned}
$$

The underlined terms in (3.12) cancel the underlined terms in (3.13) and we are left to verify that

$$
\begin{aligned}
& E_{U_{n-1}}\left(u-y_{n}\right)\left(c u y_{n} \kappa_{n-1}+\frac{1}{1+\nu^{-1} q c c_{n-1} u} \kappa_{n-1}\left(c u y_{n-1}-1\right)\right) \\
= & E_{U_{n-1}}\left(c u y_{n}-1\right)\left(-y_{n} \kappa_{n-1}+\frac{u}{u+\nu^{-1} q c_{n-1}} \kappa_{n-1}\left(u-y_{n-1}\right)\right)
\end{aligned}
$$


where, recall, $c=-q^{-1} \nu^{1}$. We use the relations $E_{U_{n-1}} y_{n-1}=E_{U_{n-1}} c_{n-1}$ and (2.22) which imply

$$
E_{U_{n-1}} y_{n} \kappa_{n-1}=E_{U_{n-1}} \kappa_{n-1} \frac{\nu^{2}}{c_{n-1}}
$$

Now the check of (3.14) is straightforward.

\subsection{Fusion procedure}

Define the fusion function $\Psi_{U_{n}}$ by

$\Psi_{U_{n}}\left(u_{1}, u_{2}, \ldots, u_{n}\right):=\left(\prod_{k=1}^{n} \frac{u_{k}-c_{k}}{c u_{k} c_{k}-1}\right) \mathcal{Y}_{1}\left(u_{1}\right) \mathcal{Y}_{2}\left(u_{1}, u_{2}\right) \cdots \mathcal{Y}_{n}\left(u_{1}, \ldots, u_{n}\right)$.

Sometimes we shall write, instead of the label $U_{n}$ of the fusion function, the corresponding sequence of contents.

The following theorem is the main result of the paper.

Theorem 3.2. The idempotent $E_{U_{n}}$ is equal to the subsequent evaluation of $\Psi_{U_{n}}$ at the points $u_{i}=c_{i}$,

$$
E_{U_{n}}=\left.\left.\Psi_{U_{n}}\left(u_{1}, u_{2}, \ldots, u_{n}\right)\right|_{u_{1}=c_{1}} \cdots\right|_{u_{n}=c_{n}}
$$

Proof. We prove the equality (3.17) again by induction on $n$. The base of induction, $n=1$, is immediate (recall that $c_{1}=1$ ):

$$
\begin{aligned}
\left.\Psi_{\square}\left(u_{1}\right)\right|_{u_{1}=c_{1}} & =\left.\frac{u_{1}-c_{1}}{c u_{1} c_{1}-1} \mathcal{Y}_{1}\left(u_{1}\right)\right|_{u_{1}=c_{1}}=\left.\frac{u_{1}-c_{1}}{c u_{1} c_{1}-1} \frac{c u_{1} c_{1}-1}{u_{1}-c_{1}}\right|_{u_{1}=c_{1}} \\
& =1=E_{U_{1}} .
\end{aligned}
$$

Assume that (3.17) is valid for the tableaux $U_{n-1}$. Then

$$
\begin{aligned}
& \left.\left.\Psi_{U_{n}}\left(u_{1}, u_{2}, \ldots, u_{n-1}, u\right)\right|_{u_{1}=c_{1}} \cdots\right|_{u_{n-1}=c_{n-1}} \\
= & \frac{u-c_{n}}{c u c_{n}-1} E_{U_{n-1}} \mathcal{Y}_{n}\left(c_{1}, \ldots, c_{n-1}, u\right) \\
= & \frac{u-c_{n}}{c u c_{n}-1} E_{U_{n-1}} \frac{c u y_{n}-1}{u-y_{n}}
\end{aligned}
$$


we used the induction hypothesis in the first equality and Lemma 3.1 in the second equality. By (3.4), the evaluation of the last expression in (3.18) at $u=c_{n}$ is equal to $E_{U_{n}}$.

Example 3.3. We shall illustrate Theorem 3.2 on the simplest example of the algebra $B M W_{2}$. The algebra $B M W_{2}$ is commutative; it is generated by $T_{1}$ which has the projector decomposition

$$
T_{1}=q S-q^{-1} A+\nu \Pi
$$

The projector $S$ is called symmetrizer - its image in the Hecke algebra quotient tends to the symmetrizer in the classical limit $q \rightarrow 1$; similarly, the projector $A$ is called antisymmetrizer. One has

$$
S=\frac{\left(T+q^{-1}\right)(T-\nu)}{\left(q+q^{-1}\right)(q-\nu)}=\frac{1}{q+q^{-1}}\left(T_{1}+q^{-1}+\frac{q-q^{-1}}{1-q \nu^{-1}} \kappa_{1}\right)
$$

$$
\begin{gathered}
A=\frac{(T-q)(T-\nu)}{\left(-q^{-1}-q\right)\left(-q^{-1}-\nu\right)}=-\frac{1}{q+q^{-1}}\left(T_{1}-q+\frac{q-q^{-1}}{1+q^{-1} \nu^{-1}} \kappa_{1}\right) \\
\Pi=\frac{(T-q)\left(T+q^{-1}\right)}{(\nu-q)\left(\nu+q^{-1}\right)}=\frac{1}{\mu} \kappa_{1}
\end{gathered}
$$

where $\mu$ is given by (2.5). The elements $S, A$ and $\Pi$ form a complete system of pairwise orthogonal primitive idempotents of $B M W_{2}$. Every element $x$ of $B M W_{2}$ is a linear combination of $S, A$ and $\Pi$; the coefficients can be found by calculating the products of $x$ with these three idempotents.

The algebra $B M W_{2}$ has three irreducible representations, all three are one-dimensional. We have three up-down tableaux with content sequences $\left\{1, q^{2}\right\},\left\{1, q^{-2}\right\}$ and $\left\{1, \nu^{2}\right\}$. The fusion function $\Psi_{\left\{c_{1}, c_{2}\right\}}$ reads

$$
\begin{aligned}
\Psi_{\left\{c_{1}, c_{2}\right\}}\left(u_{1}, u_{2}\right) & \\
& =\left(\prod_{k=1}^{2} \frac{u_{k}-c_{k}}{c u_{k} c_{k}-1}\right) \frac{c u_{1} y_{1}-1}{u_{1}-y_{1}} Q_{1}\left(u_{1}, u_{2} ; c\right) \frac{c u_{2} y_{1}-1}{u_{2}-y_{1}} T_{1}\left(u_{2}, u_{1}\right)^{-1} \\
(3.23)= & =\frac{u_{2}-c_{2}}{c u_{2} c_{2}-1} \frac{c u_{2}-1}{u_{2}-1} \frac{\left(u_{1}-u_{2}\right)^{2}}{\left(u_{2}-q^{2} u_{1}\right)\left(u_{2}-q^{-2} u_{1}\right)} T_{1}\left(\frac{1}{c u_{1} u_{2}}\right) T_{1}\left(\frac{u_{1}}{u_{2}}\right) .
\end{aligned}
$$


We used the fact that $y_{1}=c_{1}=1$. Below we write $E_{\left\{c_{1}, c_{2}\right\}}$ for the idempotent $E_{U_{2}}$ corresponding to the up-down tableau $U_{2}$ with the content sequence $\left\{c_{1}, c_{2}\right\}$.

1) Content sequence $\left\{1, q^{2}\right\}$. We have

$$
\begin{aligned}
E_{\left\{1, q^{2}\right\}} & =\left.\left.\Psi_{\left\{1, q^{2}\right\}}\left(u_{1}, u_{2}\right)\right|_{u_{1}=1}\right|_{u_{2}=q^{2}} \\
& =\frac{q \nu^{-1}+1}{q^{3} \nu^{-1}+1} \frac{q}{q+q^{-1}} T_{1}\left(-q^{-1} \nu\right) T_{1}\left(q^{-2}\right) .
\end{aligned}
$$

One finds $T_{1}\left(-q^{-1} \nu\right) \Pi=0$ and $T_{1}\left(q^{-2}\right) A=0$ so the last expression in (3.24) is proportional to the symmetrizer. Calculating $T_{1}\left(-q^{-1} \nu\right) S=q^{-1} \frac{q^{3} \nu^{-1}+1}{q \nu^{-1}+1} S$ and $T_{1}\left(q^{-2}\right) S=\left(q+q^{-1}\right) S$ we obtain

$$
E_{\left\{1, q^{2}\right\}}=S
$$

2) Content sequence $\left\{1, q^{-2}\right\}$. We have

$$
\begin{aligned}
E_{\left\{1, q^{-2}\right\}} & =\left.\left.\Psi_{\left\{1, q^{-2}\right\}}\left(u_{1}, u_{2}\right)\right|_{u_{1}=1}\right|_{u_{2}=q^{-2}} \\
& =\frac{q^{-3} \nu^{-1}+1}{q^{-5} \nu^{-1}+1} \frac{q^{-1}}{q+q^{-1}} T_{1}\left(-q^{3} \nu\right) T_{1}\left(q^{2}\right) .
\end{aligned}
$$

This time the same factor $T_{1}\left(q^{2}\right)$ is annihilated by two idempotents, $T_{1}\left(q^{2}\right) S=$ 0 and $T_{1}\left(q^{2}\right) \Pi=0$; in fact, $T_{1}\left(q^{2}\right)=-\left(q+q^{-1}\right) A$. Calculating $T_{1}\left(-q^{3} \nu\right) A=$ $-q \frac{q^{-5} \nu^{-1}+1}{q^{-3} \nu^{-1}+1} A$ we obtain

$$
E_{\left\{1, q^{-2}\right\}}=A=-\frac{1}{q+q^{-1}} T_{1}\left(q^{2}\right) .
$$

3) Content sequence $\left\{1, \nu^{2}\right\}$. We have

$$
\begin{aligned}
E_{\left\{1, \nu^{2}\right\}} & =\left.\left.\Psi_{\left\{1, \nu^{2}\right\}}\left(u_{1}, u_{2}\right)\right|_{u_{1}=1}\right|_{u_{2}=\nu^{2}} \\
& =\left.\frac{\left(u_{2}-\nu^{2}\right)\left(u_{2}-1\right)\left(q^{-1} \nu^{-1} u_{2}+1\right)}{\left(u_{2}-q^{2}\right)\left(u_{2}-q^{-2}\right)\left(q^{-1} \nu u_{2}+1\right)} T_{1}\left(-q \nu u_{2}^{-1}\right) T_{1}\left(u_{2}^{-1}\right)\right|_{u_{2}=\nu^{2}}
\end{aligned}
$$

Here the scalar coefficient has a zero at $u_{2}=\nu^{2}$, this is a new phenomenon compared to (3.24) and (3.25). The factor $T_{1}\left(u_{2}^{-1}\right)$ is regular at $u_{2}=\nu^{2}$. In the factor $T_{1}\left(-q \nu u_{2}^{-1}\right)$ the coefficient in front of $\kappa_{1}$ is $\frac{q-q^{-1}}{1-\nu^{-2} u_{2}}$ and has a simple pole at $u_{2}=\nu^{2}$; other coefficients are regular at $u_{2}=\nu^{2}$. Thus, only 
$\Pi$ survives in $T_{1}\left(-q \nu u_{2}^{-1}\right)$. Calculating $T_{1}\left(\nu^{2}\right) \Pi=\frac{\nu^{-1} q\left(q^{-1} \nu^{3}+1\right)\left(\nu-q^{-1}\right)}{\nu^{2}-1} \Pi$ we obtain

$$
E_{\left\{1, \nu^{2}\right\}}=\Pi \text {. }
$$

Example 3.4. One can rewrite the expression (3.16) for the fusion function in the form

$$
\Psi_{U_{n}}\left(u_{1}, u_{2}, \ldots, u_{n}\right)=\mathfrak{y}_{U_{n}}\left(u_{1}, u_{2}, \ldots, u_{n}\right) Y_{n}\left(u_{1}, u_{2}, \ldots, u_{n}\right)
$$

where

$$
\begin{aligned}
\mathfrak{y}_{U_{n}}\left(u_{1}, u_{2}, \ldots, u_{n}\right) & \\
& :=\prod_{k=1}^{n}\left(\frac{u_{k}-c_{k}}{c u_{k} c_{k}-1} \frac{c u_{k}-1}{u_{k}-1} \prod_{r=1}^{k-1} \frac{\left(u_{k}-u_{r}\right)^{2}}{\left(u_{k}-q^{2} u_{r}\right)\left(u_{k}-q^{-2} u_{r}\right)}\right)
\end{aligned}
$$

and

$$
\begin{aligned}
& Y_{n}\left(u_{1}, u_{2}, \ldots, u_{n}\right) \\
& \quad:=\mathcal{Q}_{2}\left(u_{1}, u_{2}\right) \cdots \mathcal{Q}_{n}\left(u_{1}, \ldots, u_{n}\right) \mathcal{T}_{n}\left(u_{1}, \ldots, u_{n}\right) \cdots \mathcal{T}_{2}\left(u_{1}, u_{2}\right)
\end{aligned}
$$

with

$$
\mathcal{Q}_{j}\left(u_{1}, \ldots, u_{j}\right):=T_{j-1}\left(\frac{1}{c u_{1} u_{j}}\right) \cdots T_{1}\left(\frac{1}{c u_{j-1} u_{j}}\right)
$$

and

$$
\mathcal{T}_{j}\left(u_{1}, \ldots, u_{j}\right):=T_{1}\left(u_{j-1}, u_{j}\right) \cdots T_{j-1}\left(u_{1}, u_{j}\right)
$$

Evaluating the numerical factors (3.28), one obtains expressions for the symmetrizer and antisymmetrizer for the algebra $B M W_{n}$ for $n>1$. The symmetrizer (its content sequence is $\left\{1, q^{2}, \ldots, q^{2(n-1)}\right\}$ ) is equal to

$$
S_{n}=\frac{q^{n(n-1) / 2}}{n_{q} !} \prod_{k=1}^{n-1} \frac{q^{2 k-1} \nu^{-1}+1}{q^{4 k-1} \nu^{-1}+1} Y_{n}\left(1, q^{2}, \ldots, q^{2(n-1)}\right)
$$

and the antiymmetrizer (its content sequence is $\left\{1, q^{-2}, \ldots, q^{2(n-1)}\right\}$ ) is equal to:

$$
A_{n}=\frac{q^{-n(n-1) / 2}}{n_{q} !} \prod_{k=1}^{n-1} \frac{q^{-2 k-1} \nu^{-1}+1}{q^{-4 k-1} \nu^{-1}+1} Y_{n}\left(1, q^{-2}, \ldots, q^{-2(n-1)}\right)
$$


Here we used the $q$-numbers, $n_{q}:=\left(q^{n}-q^{-n}\right) /\left(q-q^{-1}\right)$ and $n_{q} !:=2_{q} 3_{q} \cdots n_{q}$.

The expressions (3.32) and (3.33) can be brought to a simpler form. Indeed, by the braid relation, the product $a_{n}:=\mathcal{T}_{n}\left(1, \ldots, q^{-2(n-1)}\right) \cdots \mathcal{T}_{2}(1$, $\left.q^{-2}\right)$ (this is the part of $Y_{n}\left(1, q^{-2}, \ldots, q^{-2(n-1)}\right)$ in (3.33)) can be rewritten as a word which starts/ends by $T_{j}\left(q^{2}\right)$ for any given $j=1, \ldots, n-1$. Therefore $a_{n} T_{i}=T_{i} a_{n}=-q^{-1} a_{n}$ so $a_{n}$ is proportional to $A_{n}$. Calculating $a_{n}^{2}$ (or, equivalently, evaluating the product of the operators $\mathcal{Q}$ in (3.33) on the antisymmetrizer) one finds the proportionality coefficient and obtains the short factorized expression for the antisymmetrizer (3.33) for the algebra $B M W_{n}$ which has the same form - in terms of the baxterized elements - as the factorized expression for the antisymmetrizer for the Hecke algebra

$$
A_{n}=\frac{(-1)^{n(n-1) / 2}}{n_{q} !} \mathcal{T}_{n}\left(1, q^{-2}, \ldots, q^{-2(n-1)}\right) \cdots \mathcal{T}_{2}\left(1, q^{-2}\right)
$$

or

$$
A_{n}=\frac{(-1)^{n-1}}{n_{q}} T_{1}\left(q^{2}\right) T_{2}\left(q^{4}\right) \cdots T_{n-1}\left(q^{2(n-1)}\right) A_{n-1} .
$$

The short factorized expression for the symmetrizer is obtained by applying the isomorphism $B M W_{n}\left(-q^{-1}, \nu\right) \rightarrow B M W_{n}(q, \nu)$ defined on generators by

$$
B M W_{n}\left(-q^{-1}, \nu\right) \ni T_{j} \mapsto T_{j} \in B M W_{n}(q, \nu), j=1, \ldots, n-1
$$

The replacement $q \mapsto-q^{-1}$ in (2.15) and (2.19) produces the second set $T_{i}^{*}(u, v)$ and $Q_{i}^{*}(u, v ; c)$ of baxterized elements and converts (3.34), (3.35) to the short factorized expression for the symmetrizer (3.32)

$$
\begin{aligned}
S_{n}= & \frac{1}{n_{q}} T_{1}^{*}\left(q^{-2}\right) T_{2}^{*}\left(q^{-4}\right) \cdots T_{n-1}^{*}\left(q^{-2(n-1)}\right) S_{n-1} \\
= & \frac{1}{n_{q} !}\left[T_{1}^{*}\left(q^{-2}\right) T_{2}^{*}\left(q^{-4}\right) \cdots T_{n-1}^{*}\left(q^{-2(n-1)}\right)\right]\left[T_{1}^{*}\left(q^{-2}\right) T_{2}^{*}\left(q^{-4}\right) \cdots\right. \\
& \left.\cdots T_{n-2}^{*}\left(q^{-2(n-2)}\right)\right] \cdots T_{1}^{*}\left(q^{-2}\right)
\end{aligned}
$$

The construction of all idempotents can be equivalently done with the help of the elements $T_{i}^{*}(u, v)$ and $Q_{i}^{*}(u, v ; c)$; the substitution $q \mapsto-q^{-1}$ into the expression for the $E_{U_{n}}$ leads to the idempotent $E_{U_{n}^{t}}$ for the transposed updown tableau. 
The formulas (3.35) and (3.36) for the symmetrizer and antisymmetrizer were suggested in [10], see also [16]. We refer the reader to the works [6], [9] and [36], where the symmetrizer and antisymmetrizer are given in several other forms.

\subsection{Hecke algebra}

The fusion functions (3.16) for the BMW algebra depend on $c$, whose value is related to the parameter $\nu$ by (3.5). In the Hecke algebra quotient of the BMW algebra (the quotient by the ideal generated by all $\kappa_{i}$ ) the parameter $\nu$ disappears. However the images of the fusion functions $\Psi_{U_{n}}$ in the Hecke algebra quotient still contain non-trivially the parameter $c$. Considering $c$ as a free parameter in the Hecke algebra quotient we obtain a one parametric family of fusion functions (for the Hecke algebras), given by (3.16), where we have to substitute

$$
T_{i}\left(u_{1}, u_{2}\right)=T_{i}+\frac{q-q^{-1}}{u_{2} / u_{1}-1}, \quad Q_{i}\left(u_{1}, u_{2} ; c\right)=T_{i}+\frac{q-q^{-1}}{c u_{1} u_{2}-1}
$$

in the definition (3.7). Explicitly the one-parametric family of the fusion functions for the Hecke algebra reads

$$
\begin{aligned}
& \widetilde{\Psi}_{U_{n}}\left(u_{1}, u_{2}, \ldots, u_{n}\right) \\
:= & \left(\prod_{k=1}^{n} \frac{u_{k}-c_{k}}{c u_{k} c_{k}-1} \frac{c u_{k}-1}{u_{k}-1}\right) \widetilde{Y}_{1}\left(u_{1}\right) \widetilde{Y}_{2}\left(u_{1}, u_{2}\right) \cdots \widetilde{Y}_{n}\left(u_{1}, \ldots, u_{n}\right),
\end{aligned}
$$

where

$$
\tilde{Y}_{j}\left(u_{1}, \ldots, u_{j}\right):=\prod_{k: j>k \geqslant 1}\left(T_{k}+\frac{q-q^{-1}}{c u_{k} u_{j}-1}\right) \prod_{k: 1 \leqslant k<j}\left(T_{k}+\frac{q-q^{-1}}{u_{j} / u_{k}-1}\right)^{-1}
$$

$j=1,2, \ldots ;$ the empty product is equal to 1 , the symbol $\longleftarrow$ (respectively, $\longrightarrow$ ) over $\prod$ means that the product is ordered according to the descend (respectively, ascend) of the product index $k$.

The fusion function (3.37) generalizes the fusion function proposed in [13]; the results of [13] are reproduced at $c=0$ since $Q_{i}\left(u_{1}, u_{2} ; 0\right)=T_{i}^{-1}$.

In the classical limit $q \rightarrow 1$, the family (3.37) of fusion functions leads to the one-parametric family, discussed in [12], of fusion functions for the symmetric group. 


\subsection{Reflection equation and fusion functions}

It was discovered in [15] that the element

$$
L_{j}(u)=\frac{c u y_{j}-1}{u-y_{j}}
$$

solves the reflection equation

$$
L_{j}(u) T_{j}\left(\frac{1}{c u v}\right) L_{j}(v) T_{j}\left(\frac{u}{v}\right)=T_{j}\left(\frac{u}{v}\right) L_{j}(v) T_{j}\left(\frac{1}{c u v}\right) L_{j}(u)
$$

in the BMW algebra. The element (3.39) is just the same as in (3.8). This coincidence is important in the study of the evaluation homomorphisms for the quantum universal enveloping algebras, see [12] for the classical counterpart.

The main ingredients of the fusion function - the elements $\mathcal{Y}_{j}, j=$ $1, \ldots, n-1$, defined in $(3.7)$ - also satisfy the reflection equation

$$
\begin{aligned}
& \mathcal{Y}_{j}\left(u_{1}, \ldots, u_{j-1}, v\right) T_{j}\left(\frac{1}{c u v}\right) \mathcal{Y}_{j}\left(u_{1}, \ldots, u_{j-1}, u\right) T_{j}\left(\frac{u}{v}\right)^{-1} \\
= & T_{j}\left(\frac{u}{v}\right)^{-1} \mathcal{Y}_{j}\left(u_{1}, \ldots, u_{j-1}, u\right) T_{j}\left(\frac{1}{c u v}\right) \mathcal{Y}_{j}\left(u_{1}, \ldots, u_{j-1}, v\right) .
\end{aligned}
$$

This is shown by induction on $j$.

The reflection equation (3.41) can be represented graphically as

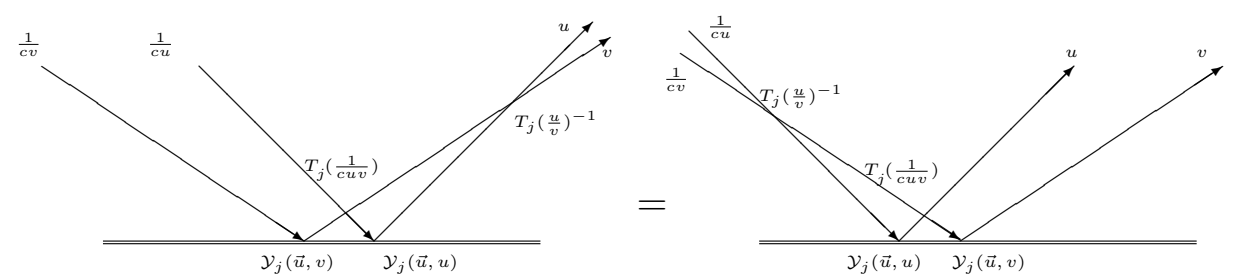

Figure 1

Here we used the notation $\vec{u}:=\left\{u_{1}, \ldots, u_{j-1}\right\}$. This picture gives the standard physical interpretation of the reflection equation (3.41) which is used in the theory of the factorized scattering of particles on the half-line, see $[4],[7]$. The reflection from the boundary is described by the operator $\mathcal{Y}_{j}$ while the collision of two particles is described by the operator $T_{j}$ or $T_{j}^{-1}$. The left hand side of the equality in Figure 1 represents the process when: first, the particle with the spectral parameter $\frac{1}{c v}$ reflects from the boundary and changes its spectral parameter to $v$; second, this particle collides with 
the particle having the spectral parameter $\frac{1}{c u}$; third, the particle with the spectral parameter $\frac{1}{c u}$ reflects from the boundary and acquires the spectral parameter $u$; finally the two reflected particles collide. Algebraically this sequence of events is given by the left hand side of (3.41). The picture in the right hand side of the equality in Figure 1 shows the different scenario of the same process (with different initial positions of the particles) and algebraically is expressed by the right hand side of (3.41). By the factorizability of the scattering both scenarios lead to the same result which is expressed by the Equation (3.41).

One can write the fusion function (3.16) in the form (cf. (3.27)):

$$
\begin{aligned}
& \Psi_{U_{n}}\left(u_{1}, u_{2}, \ldots, u_{n}\right) \\
= & \left(\prod_{k=1}^{n} \frac{u_{k}-c_{k}}{c u_{k} c_{k}-1}\right) \mathfrak{Q}_{n}\left(u_{1}, \ldots, u_{n}\right) \mathcal{T}_{n}^{\prime}\left(u_{1}, \ldots, u_{n}\right) \cdots \mathcal{T}_{2}^{\prime}\left(u_{1}, u_{2}\right),
\end{aligned}
$$

where

$$
\mathcal{T}_{j}^{\prime}\left(u_{1}, \ldots, u_{j}\right):=T_{1}\left(u_{j}, u_{j-1}\right)^{-1} \cdots T_{j-1}\left(u_{j}, u_{1}\right)^{-1}
$$

$$
\mathfrak{Q}_{n}\left(u_{1}, \ldots, u_{n}\right):=\mathcal{Y}_{1}\left(u_{1}\right) \mathcal{Q}_{2}\left(u_{1}, u_{2}\right) \mathcal{Y}_{1}\left(u_{2}\right) \cdots \mathcal{Q}_{n}\left(u_{1}, \ldots, u_{n}\right) \mathcal{Y}_{1}\left(u_{n}\right)
$$

with $\mathcal{Q}_{j}\left(u_{1}, \ldots, u_{j}\right)$ given by $(3.30)$.

Graphically, the part

$$
\mathfrak{Q}_{n}\left(u_{1}, \ldots, u_{n}\right) \mathcal{T}_{n}^{\prime}\left(u_{1}, \ldots, u_{n}\right) \cdots \mathcal{T}_{2}^{\prime}\left(u_{1}, u_{2}\right)
$$

of the fusion function (3.42) is represented as the process of scattering of $n$ particles on the half-line. For example, the expression (3.45) for $n=4$ (3.46)

$$
\begin{gathered}
\mathcal{Y}_{1}\left(u_{1}\right) \mathcal{Q}_{2}\left(u_{1}, u_{2}\right) \mathcal{Y}_{1}\left(u_{2}\right) \mathcal{Q}_{3}\left(u_{1}, u_{2}, u_{3}\right) \mathcal{Y}_{1}\left(u_{3}\right) \mathcal{Q}_{4}\left(u_{1}, u_{2}, u_{3}, u_{4}\right) \mathcal{Y}_{1}\left(u_{4}\right) \cdot \\
\mathcal{T}_{4}^{\prime}\left(u_{1}, u_{2}, u_{3},, u_{4}\right) \mathcal{T}_{3}^{\prime}\left(u_{1}, u_{2}, u_{3}\right) \mathcal{T}_{2}^{\prime}\left(u_{1}, u_{2}\right)
\end{gathered}
$$

is visualized in Figure 2: 


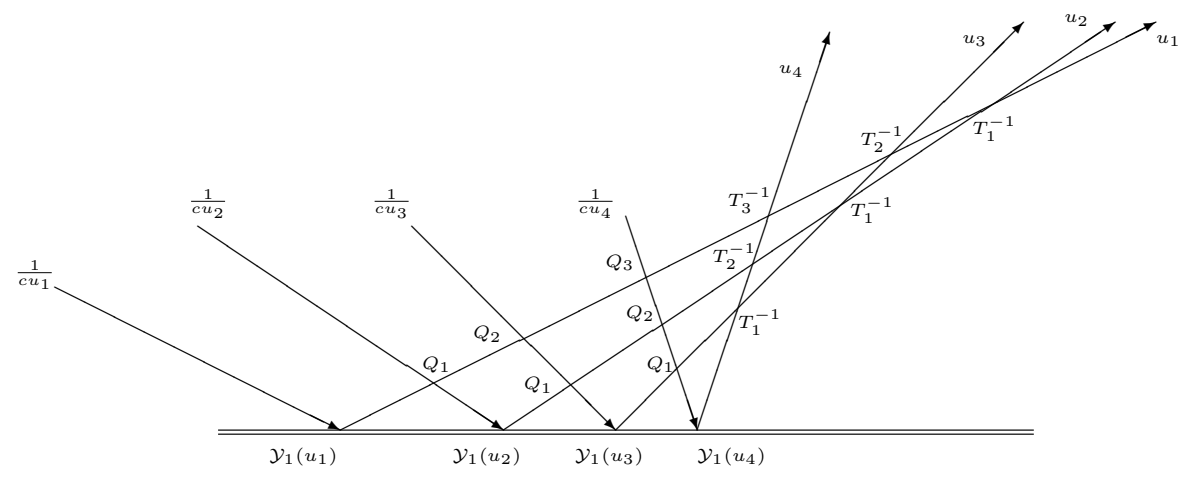

Figure 2

In Figure 2 the spectral parameters for operators $T_{k}^{-1}$ and $Q_{k}$ can be restored by the rules:

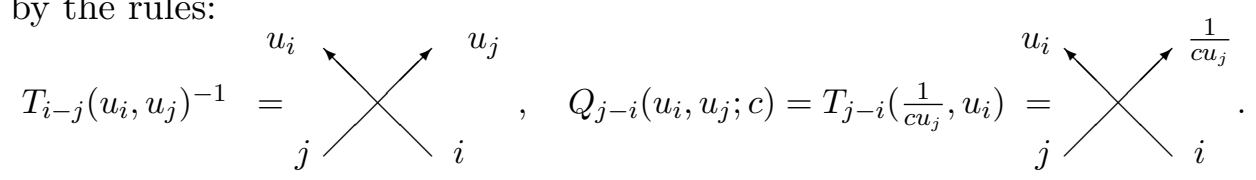

Remark. There is another interpretation of the expressions (3.45) for different $n$. Namely these are fusion solutions of the so-called higher reflection equations. The fusion method was proposed in [21],[22] for the Yang-Baxter equation and in [24] for the reflection equation. The interpretation of (3.45) as a fusion solution of reflection equation justifies the name "fusion function" for $\Psi_{U_{n}}$ in (3.16).

\section{Classical limit}

In this section we demonstrate that the fusion procedures of [11] and [12] for the Brauer algebra can be obtained from the fusion procedure for the BMW algebra by taking two classical limits. Following [12], we denote the generators of the Brauer algebra $\mathcal{B}_{n}(\omega)$ by $s_{1}, \ldots, s_{n-1}, \epsilon_{1}, \ldots, \epsilon_{n-1}$. However, we denote by $\theta_{i}$ the rational spectral parameters instead of the $u_{i}$ used in [12] to avoid confusion.

The symbol $\rightarrow$ in this Section means "tends to".

\subsection{Contraction of the BMW algebra to the Brauer algebra}

The BMW algebra is a flat deformation of the Brauer algebra; expressed differently, the Brauer algebra is a contraction of the BMW algebra. To 
describe the contraction, we set

$$
q=e^{h}, \quad \nu=q^{1-\omega}=e^{h(1-\omega)}
$$

and let $h$ tend to 0 ,

$$
h \rightarrow 0
$$

In the contraction, the generator $T_{i}$ tends to $s_{i}$ which has order 2 ; in the limit (4.2) the element $\kappa_{i}$ becomes thus an independent generator; we have

$$
T_{i} \rightarrow s_{i}, \quad \kappa_{i} \rightarrow \epsilon_{i}
$$

The expression (4.1) for $\nu$ contains $\omega$ which becomes the parameter of the Brauer algebra family.

\subsection{Behavior of the spectral parameters}

The description of the contraction is given by the formulas (4.1), (4.2) and (4.3). However the fusion function for the BMW algebra depends on the spectral parameters $u_{j}$ and we have to complete the formulas (4.1), (4.2) and (4.3) for the contraction with the information about the behavior of the spectral parameters; the relation to the rational spectral parameters is given by

$$
u_{j}=e^{2 h\left(\theta_{j}-\frac{\omega-1}{2}\right)}
$$

for the classical limit, determined by (4.1), (4.2) and (4.3).

To explain the prescription (4.4) recall the definition of the classical content of boxes in an up-down tableau $U_{n}=\left(\Lambda_{1}, \Lambda_{2}, \ldots, \Lambda_{n}\right)$. For the box in the $a$-th row and $b$-th column the classical content is defined by

$$
(b-a)+\frac{\omega-1}{2}
$$

if this box was added to the diagram $\Lambda_{j-1}$ to obtain the diagram $\Lambda_{j}$ and

$$
-(b-a)-\frac{\omega-1}{2}
$$

if this box was removed from the diagram $\Lambda_{j-1}$ to obtain the diagram $\Lambda_{j}$.

The quantum content is defined in (3.2) and (3.3). In (4.4) we made the shift in the exponent for the agreement between the quantum and classical contents. 


\subsection{Classical limit of the fusion function}

Performing the classical limit, determined by (4.2), (4.1), (4.3) and (4.4), of the building blocks $Q(u, v)$ and $T(u, v)$ of the fusion function, we find

$$
\begin{aligned}
& Q_{i}\left(u_{1}, u_{2}\right) \rightarrow s_{i}-\frac{\epsilon_{i}}{\theta_{1}+\theta_{2}}=s_{i}\left(1-\frac{\epsilon_{i}}{\theta_{1}+\theta_{2}}\right), \\
& T_{i}\left(u_{1}, u_{2}\right) \rightarrow s_{i}-\frac{1}{\theta_{1}-\theta_{2}}=s_{i}\left(1-\frac{s_{i}}{\theta_{1}-\theta_{2}}\right),
\end{aligned}
$$

so this classical limit of (3.16) reproduces the fusion function from [11].

\subsection{Second contraction}

There exists a different contraction of the BMW algebra to the Brauer algebra. It is determined by $(4.2),(4.3)$, and

$$
q=-e^{h}, \quad \nu=e^{h(\omega-1)}
$$

instead of (4.1).

\subsection{Behavior of the spectral parameters for the second contraction}

As we shall see, in the limit determined by (4.2), (4.8) and (4.3), we obtain the idempotents for the transposed diagrams and up-down tableaux. This phenomenon is clearly illustrated on the example of the (anti)symmetrizers. Indeed, on the $q$-deformed level, the symmetrizer is the projector on the eigenspace with the eigenvalue $q$. With the prescription (4.2) the parameter $q$ tends to $(-1)$ so the $q$-symmetrizer becomes the classical antisymmetrizer in this limit.

With the prescription (4.8), the quantum and classical contents cannot be made compatible within the Ansatz $u=e^{h f(\theta)}$ with any function $f(u$ is the quantum content, $\theta$ is the classical one); the definition of the classical content of a box has to be modified. Following the above example of the (anti)symmetrizers, we define the t-classical content of a box to be the usual classical content of the transposed box (" $\mathrm{t}$ " in t-classical stands for transposition). We shall write explicitly the analogues of the formulas (4.5) and (4.6) for an up-down tableau $U_{n}=\left(\Lambda_{1}, \Lambda_{2}, \ldots, \Lambda_{n}\right)$. For the box in the $a$-th 
row and $b$-th column the t-classical content is defined by

$$
-(b-a)+\frac{\omega-1}{2}
$$

if this box was added to the diagram $\Lambda_{j-1}$ to obtain the diagram $\Lambda_{j}$ and

$$
(b-a)-\frac{\omega-1}{2}
$$

if this box was removed from the diagram $\Lambda_{j-1}$ to obtain the diagram $\Lambda_{j}$.

Instead of (4.4) we impose now

$$
u_{j}=e^{2 h\left(-\theta_{j}+\frac{\omega-1}{2}\right)}
$$

for the agreement of the quantum and t-classical contents.

\subsection{Second classical limit of the fusion function}

Performing the classical limit, determined by (4.2), (4.8), (4.3) and (4.11), of the elements $Q(u, v)$ and $T(u, v)$, we find

$$
\begin{aligned}
& Q_{i}\left(u_{1}, u_{2}\right) \rightarrow s_{i}+\frac{1}{\theta_{1}+\theta_{2}-\varkappa}-\frac{\epsilon_{i}}{\theta_{1}+\theta_{2}}=s_{i}\left(1+\frac{s_{i}}{\theta_{1}+\theta_{2}-\varkappa}-\frac{\epsilon_{i}}{\theta_{1}+\theta_{2}}\right), \\
& T_{i}\left(u_{1}, u_{2}\right) \rightarrow s_{i}-\frac{1}{\theta_{1}-\theta_{2}}+\frac{e_{i}}{\theta_{1}-\theta_{2}-\varkappa}=s_{i}\left(1-\frac{s_{i}}{\theta_{1}-\theta_{2}}+\frac{e_{i}}{\theta_{1}-\theta_{2}-\varkappa}\right),
\end{aligned}
$$

where $\varkappa=\frac{\omega}{2}-1$. Thus the function (3.16) in this limit tends precisely to the fusion function introduced in [12].

We repeat that the evaluation of the fusion function (3.16) for the BMW algebra on contents of an up-down tableau $U_{n}$ descends to the evaluation of the limiting fusion function for the Brauer algebra on the transposed tableau $U_{n}^{T}$. The idempotents belonging to the diagram $\lambda$ of the BMW algebra tend to the idempotents belonging to the diagram $\lambda^{T}$ of the Brauer algebra.

\subsection{Further contractions}

In the two contractions above, $q$ was tending to 1 or $(-1)$. When we fix the behavior of $q$ there are two possibilities for the behavior of $\nu$ : (2.5), considered as an equation for $\nu$, is quadratic and we must have $\mu \rightarrow \omega$ in the classical limit. Therefore, the BMW-algebra contracts to the Brauer 
algebra in two more regimes ( $h \rightarrow 0$ as before):

$$
q=e^{h}, \quad \nu=-e^{h(\omega-1)}
$$

and

$$
q=-e^{h}, \quad \nu=-e^{h(1-\omega)}
$$

For these regimes we have to impose that the generator $T_{i}$ tends to $\left(-s_{i}\right)$ in the classical limit to respect the relations $s_{i} \epsilon_{i}=\epsilon_{i} s_{i}=\epsilon_{i}$ of the Brauer algebra. Thus, (4.3) gets replaced by

$$
T_{i} \rightarrow-s_{i}, \quad \kappa_{i} \rightarrow \epsilon_{i}
$$

We omit further details of the corresponding limits of the fusion function (3.16).

\section{Acknowledgement}

The work of API was supported by the Russian Science Foundation, Grant No. 14-11-00598.

\section{References}

[1] A. Beliakova and C. Blanchet, Skein construction of idempotents in Birman-Murakami-Wenzl algebras. Math. Ann. 321 no. 2 (2001) 347 -373 .

[2] J. Birman and H. Wenzl, Braids, link polynomials and a new algebra. Trans. Amer. Math. Soc. 313 (1989) 249-273.

[3] Y. Cheng, M. L. Ge and K. Xue, Yang-Baxterization of braid group representations. Comm. Math. Phys. 136 no. 1 (1991) 195-208.

[4] I. V. Cherednik, Factorizing particles on a half-line and root systems. Theor. Math. Phys., 61 no. 1 (1984) 977-983.

[5] I. V. Cherednik, A new interpretation of Gelfand-Tzetlin bases. Duke Math. J. 54 no. 2 (1987) 563-577.

[6] R. Dipper, Jun Hu, F. Stoll, Symmetrizers and antisymmetrizers for the BMW algebra. arXiv: 1109.0342. 
[7] S. Ghoshal, A. B. Zamolodchikov, Boundary S-Matrix and boundary state in two-dimensional integrable quantum field theory. Int. J. Mod. Phys. A9 (1994) 3841-3886; arXiv:hep-th/9306002

[8] F. M. Goodman, P. de la Harpe and V. F. R. Jones, Coxeter graphs and towers of algebras, Springer (1989).

[9] I. Heckenberger, A. Schüler, Symmetrizer and antisymmetrizer of the Birman-Wenzl-Murakami algebras. Lett. Math. Phys., 50 (1) (1999) $45-51$.

[10] A. P. Isaev, Quantum groups and Yang-Baxter equations. preprint MPIM (Bonn), MPI 2004-132 (2004), http://webdoc.sub.gwdg.de/ebook/serien/e/mpi_mathematik/200 4/132.pdf

[11] A. P. Isaev and A. I. Molev, Fusion procedure for the Brauer algebra. Algebra i Analiz 22 no. 3 (2010) 142-154; arXiv:0812.4113 [math.RT]

[12] A. P. Isaev, A. I. Molev and O. V. Ogievetsky, A new fusion procedure for the Brauer algebra and evaluation homomorphisms. Int. Math. Res. Not. 2011 doi: 10.1093/imrn/rnr126; arXiv:1101.1336 [math.RT]

[13] A. P. Isaev, A. I. Molev and A. F. Os'kin, On the idempotents of Hecke algebra. Lett. Math. Phys. 85 (2008) 79-90; arXiv:0804.421 [math.QA]

[14] A. P. Isaev and O. V. Ogievetsky, On representations of Hecke algebras. Czech. Journ. Phys. 55 no. 11 (2005) 1433-1441.

[15] A. P. Isaev and O. V. Ogievetsky, On Baxterized solutions of reflection equation and integrable chain models. Nucl. Phys. B 760 (2007) 167183; arXiv:math-ph/0510078

[16] A. P. Isaev and O. V. Ogievetsky, Braids, shuffles and symmetrizers. J. Phys. A: Math. Theor. 42 (2009) 1-15; arXiv:math.QA/0511618

[17] A. P. Isaev and O. Ogievetsky, Jucys-Murphy elements for BirmanMurakami-Wenzl algebras. Physics of Particles and Nuclei Letters 8 no. 3 (2011) 394-407; arXiv:0912.4010 [math.QA]

[18] V. F. R. Jones, On a certain value of the Kauffman polynomial. Comm. Math. Phys. 125 (1989) 459-467. 
[19] A. Jucys, On the Young operators of the symmetric group. Lietuvos Fizikos Rinkinys 6 (1966) 163-180.

[20] A. Jucys, Factorization of Young projection operators for the symmetric group. Lietuvos Fizikos Rinkinys 11 (1971) 5-10.

[21] P. P. Kulish, N. Yu. Reshetikhin and E. K. Sklyanin, Yang-Baxter equation and representation theory: I. Lett. in Math. Phys. 5 no. 5 (1981) 393-403.

[22] P. P. Kulish and E. K. Sklyanin, Quantum spectral transform method: recent developments. In: Integrable Quantum Field Theories, Lect. Notes Phys. 151 (1982) 61-119.

[23] R. Leduc and A. Ram, A ribbon Hopf algebra approach to the irreducible representations of centralizer algebras: The Brauer, Birman-Wenzl, and type A Iwahori-Hecke algebras. Advances in Mathematics 125 no. 1 (1997) 1-94.

[24] L. Mezinchescu and R. Nepomechie, Fusion procedure for open chains. J. Phys. A: Math. Gen. 25 (1992) 2533-2543.

[25] A. I. Molev, On the fusion procedure for the symmetric group. Rep. Math. Phys. 61 (2008) 181-188; arXiv:math/0612207 [math.RT]

[26] J. Murakami, The Kauffman polynomial of links and representation theory. Osaka J. Math. 24 (1987) 745-758.

[27] J. Murakami, Solvable lattice models and algebras of face operators. Adv. Studies in Pure Math. 19 (1989) 399-415.

[28] J. Murakami, The representation of the q-analogue of Brauer's centralizer algebras and the Kauffman polynomial of links. Publ. RIMS 26 no. 6 (1990) 935-945.

[29] G. E. Murphy, A new construction of Young's seminormal representation of the symmetric groups. J. Algebra 69 (1981) 287-297.

[30] M. Nazarov, Yangians and Capelli identities. In: Kirillov's Seminar on Representation Theory (G. I. Olshanski, ed.), Amer. Math. Soc. Transl. 181, Amer. Math. Soc., Providence, RI (1998) 139-163.

[31] M. Nazarov, A mixed hook-length formula for affine Hecke algebras. European J. Combin. 25 (2004) 1345-1376.

[32] O. V. Ogievetsky, Uses of quantum spaces. Contemp. Math. 294, Amer. Math. Soc., Providence, RI (2002) 161-232. 
[33] O. Ogievetsky and L. Poulain d'Andecy, On representations of cyclotomic Hecke algebras. Mod. Phys. Lett. A 26 no. 11 (2011) 795-803; arXiv:1012.5844 [math-ph]

[34] A. Okounkov and A. Vershik, A new approach to representation theory of symmetric groups II. Selecta Math (New series) 2 no. 4 (1996) 581605 .

[35] A. Ram and H. Wenzl, Matrix units for centralizer algebras. J. Algebra 145 no. 2 (1992) 378-395.

[36] I. Tuba, H. Wenzl, On braided tensor categories of type BCD. J. Reine Angew. Math. 581 (2005) 31-69.

[37] H. Wenzl, Quantum groups and subfactors of type B, C and D. Comm. Math. Phys. 133 (1990) 383-432.

Bogoliubov Laboratory of Theoretical Physics

Joint Institute for NuClear Research

Dubna, Moscow Region 141980, Russia

E-mail address: isaevap@theor.jinr.ru

School of Mathematics And Statistics

University of Sydney, NSW 2006, Australia

E-mail address: alexander.molev@sydney.edu.au

Center of Theoretical Physics ${ }^{2}$, Luminy

13288 Marseille, France

AND

J.-V. Poncelet French-Russian Laboratory, UMI 2615 du CNRS

INDEPENDENT UNIVERSITY OF MOSCOW

11 B. Vlasievski Per., 119002 Moscow, Russia

E-mail address: oleg@cpt.univ-mrs.fr

\footnotetext{
${ }^{2}$ Unité Mixte de Recherche (UMR 6207) du CNRS et des Universités AixMarseille I, Aix-Marseille II et du Sud Toulon - Var; laboratoire affilié à la FRUMAM (FR 2291)
} 
